\title{
Concreto de alto rendimiento, predicción de su resistencia a la compresión mediante redes neuronales artificiales
}

\section{High performance concrete, prediction of its resistance to compression through artificial neuronal networks}

\author{
Luis Acuña', Pedro Espinoza', Isabel Moromi ${ }^{2}$, Ana Torre², \\ Francisco García ${ }^{3}$ \\ ${ }^{1}$ Facultad de Ingeniería Industrial y de Sistemas, Universidad Nacional de Ingeniería. Av. Túpac Amaru, 210. Lima - Perú. \\ ${ }^{2}$ Facultad de Ingeniería Civil, Universidad Nacional de Ingeniería. Av. Túpac Amaru, 210. Lima - Perú. \\ ${ }^{3}$ Dpto. Ingeniería Forestal. Universidad Politécnica de Madrid. Ciudad Universitaria S/N, 28040 Madrid - España,
}

Recibido: 15/01/2017 Aceptado: 25/04/2017

\section{RESUMEN}

En las edificaciones de las viviendas modernas el concreto es un elemento fundamental que interviene. De otro lado en las construcciones de puentes, diques, túneles, esto es en la construcción de estructuras no estándares de la ingeniería civil, el concreto que se utiliza es el de alto rendimiento (CAR) que aparte de los componentes básicos como el agua, cemento, agregados finos y gruesos, contienen otros aditivos cementantes, como las microsílices. El problema es conseguir un recurso tecnológico que ayude a pronosticar la resistencia de CAR a partir de sus datos de fabricación, pero esto es imposible. Sin embargo, se tiene las redes neuronales artificiales que cumplen este papel, que luego de entrenadas se transforman en verdaderas funciones matemáticas que aproximan los valores esperados de las resistencias de las probetas de concreto. El nivel de aproximación se estima por la correlación entre la respuesta y el valor esperado de la red. Entonces resulta muy útil contar con una red neuronal que permita simular numéricamente la resistencia del concreto, incluso antes de su fabricación. En esta investigación se han obtenido diversas redes neuronales artificiales que pronostican la resistencia a compresión del CAR con correlaciones que varían entre 0.86 y 0.91 .

Palabras Clave: Red Neuronal Artificial, probeta, compresión axial, aditivos.

\section{ABSTRACT}

The building of modern housing concrete is a fundamental element that intervenes. On the other hand, in the construction of bridges, dams, tunnels, this is in the construction of non-standard civil engineering structures, the concrete that is used is the high performance (CAR) that apart from the basic components such as water, Cement, fine and coarse aggregates, contain other cementing additives, such as microsílices. The problem is to get a technological resource that helps predict the resistance of CAR from its manufacturing data, but this is impossible. However, we have artificial neural networks that fulfill this role, which after being transformed into true mathematical functions that approximate the expected values ??of the resistance of concrete specimens. The approximation level is estimated by the correlation between the response and the expected value of the network. It is then very useful to have a neural network that simulates numerically the resistance of the concrete, even before its manufacture. In this investigation, several artificial neural networks have been obtained that predict the resistance to compression of the CAR with correlations that vary between 0.86 and 0.91 .

Keywords: Artificial Neural Network, test tube, axial compression, additives

\section{INTRODUCCIÓN}

Las 500 probetas de concreto han sido elaboradas por el Laboratorio de Ensayo de Materiales (LEM) de la Facultad de Ingeniería Civil de la UNI. Las elabora con diferentes valores de sus componentes de fabricación (Fig.1) siguiendo la norma internacional ASTM C 192/C $192 \mathrm{M}$ y luego de un tiempo de curado mide la resistencia a la compresión axial (Fig. 2) siguiendo la norma internacional ASTM, C39/C 39M.

Correspondencia:

francisco.garcia@upm.es

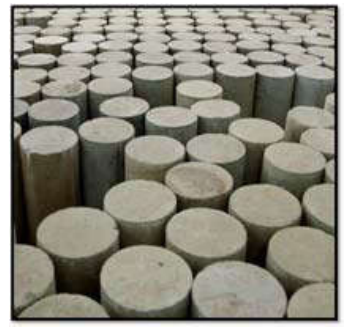

Figura 1. Probetas de concreto

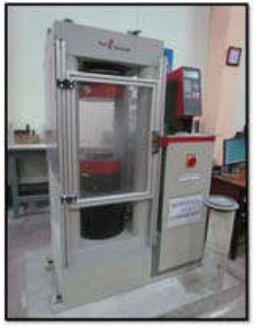

Figura 2. Máquina que mide la resistencia 
Así se tiene una base de datos que registra los valores de las variables de fabricación y la resistencia de 500 probetas de concreto. Luego de un depurado se ha reducido a 10 variables de fabricación y una que es la resistencia, como se muestra en la siguiente tabla.

Tabla 1. Variables Usadas

\begin{tabular}{|c|l|}
\hline $\mathbf{N}^{\circ}$ & \multicolumn{1}{|c|}{ Variables } \\
\hline 1 & Identificación de muestras \\
\hline 2 & Área $\left(\mathrm{cm}^{2}\right)$ \\
\hline 3 & Aditivo \\
\hline 4 & $\%$ De aditivo (material cementante) \\
\hline 5 & Aditivo $(\mathrm{kg} / \mathrm{m} 3)$ \\
\hline 6 & $\%$ De microsílice (material cementante) \\
\hline 7 & Microsílice $(\mathrm{kg} / \mathrm{m} 3)$ \\
\hline 8 & Relación a/c \\
\hline 9 & Cemento $(\mathrm{kg} / \mathrm{m} 3)$ \\
\hline 10 & Arena $(\mathrm{kg} / \mathrm{m} 3)$ \\
\hline 11 & Resistencia a la compresión $\left(\mathrm{kg} / \mathrm{cm}^{2}\right)$ \\
\hline
\end{tabular}

\section{EL PROBLEMA}

Es conseguir un sistema que aproxime la resistencia de una probeta partiendo de los datos de fabricación. Esto se consigue con las Redes Neuronales Artificiales (RNA) Supervisadas.

Con esta finalidad se hará una exposición de la noción de redes neuronales, de su aprendizaje y de su capacidad de aproximar funciones de muchas variables empleando técnicas de minimización de funciones de muchas variables pero que caen en el universo de los problemas de mínimos cuadrados. Entre estas se encuentran los métodos iterativos basados en el método de Levenberg-Marquardt. Para evaluar la respuesta de una Red Neuronal Artificial se utiliza el coeficiente de regresión (R), el coeficiente de determinación (R2), el error medio cuadrático (MSE) y la raíz del error cuadrático medio (RMSE).

Existen otros modelos que tratan de explicar el endurecimiento del concreto como el de [10] Martinelli et. al que se basa en una Ecuación Diferencial en Derivadas Parciales de evolución con datos iniciales y de frontera.

$$
\begin{aligned}
& \frac{\partial T}{\partial t}(x, t)=c * \frac{\partial^{2} T}{\partial x^{2}}(x, t)+d^{*} e^{-a\left(\frac{b-T(x, t)}{b^{*} T(x, t)}\right)} \\
& \text { con } 0<x<L ; t>0 \\
& T(x, 0)=\alpha(x) ; T(0, t)=f(t) ; T(L, t)=g(t)
\end{aligned}
$$

Este modelo viene a ser una ecuación diferencial de tipo Elíptico Semilineal Evolutivo.

\section{MATERIAL Y MÉTODOS}

En el presente trabajo se han utilizado 500 probetas de concreto fabricado con distintos tipos y cantidades de cemento, arena, piedra, agua, aditivos y microsílices. Para esto se ha seguido la norma ASTM C 192/C 192M [1]. Los ensayos de compresión se han realizado según la norma ASTM C39/C 39M [2] con diferentes periodos de curado.

\section{Aditivos}

Los aditivos son ingredientes del concreto que se adicionan a la mezcla inmediatamente antes o durante el mezclado.

Las razones principales para el uso de aditivos son:

a. Reducción del costo de la construcción de concreto.

b. Obtención de ciertas propiedades en el concreto de manera más efectiva que otras.

c. Manutención de la calidad del concreto durante las etapas de mezclado, transporte, colocación y curado en condiciones de clima adverso.

d. Superación de ciertas emergencias durante las operaciones de mezclado, transporte específicamente para concretos de baja relación a/ c, autoconsolidados y autonivelantes, empleado en climas fríos y templados.

\section{Aplicaciones:}

a. Como hiperplastificante: Proporciona a la mezcla del concreto un incremento en el asentamiento (slump) sin necesidad de agregar más agua, facilitando la colocación del mismo haciéndolo apto para el bombeo.

b. Como reductor de agua de alto rango: Permite reducir hasta aproximadamente un 35\% de agua logrando obtener un concreto con trabajabilidad, impermeabilidad y con altas resistencias en todas las edades (Resistencia a la Compresión)

c. Como ahorrador de cemento: Al disminuir la cantidad de agua Relación a/c se consigue un incremento en las resistencias. Al reducir cemento manteniendo la relación $\mathrm{a} / \mathrm{c}$ inicial se consiguen iguales o superiores resistencias a las diseñadas según patrón reduciendo el costo por metro cúbico de concreto, según Tabla 2.

Tabla 2. Ahorrador cemento

\begin{tabular}{|l|l|}
\hline Apariencia & Líquido \\
\hline Color & Ámbar \\
\hline Densidad & $1.05 \mathrm{~kg} / \mathrm{l}$ \\
\hline Solubilidad & Soluble en agua \\
\hline
\end{tabular}


En la presente investigación se utilizará porcentajes de $2.5 \%$ a $1.5 \%$ del peso del material cementante dependiendo de la relación $\mathrm{a} /(\mathrm{c}+\mathrm{p})$ a usar.

\section{Microsílice}

La microsílice es un subproducto que se usa como una puzolana. Este subproducto es el resultado de la reducción del cuarzo de alta pureza con carbón en hornos eléctricos durante la producción de liga de silicio o ferrosilicio. La microsílice sube como un vapor oxidado de los hornos a $2000^{\circ} \mathrm{C}\left(3620^{\circ} \mathrm{F}\right)$. Cuando se enfría, el humo se condensa y se colecta en bolsas de tela enormes. La microsílice condensada se procesa para removerle las impurezas y para controlar el tamaño de las partículas.

Se trata de un material extremamente fino, con partículas con menos de $1 \mu \mathrm{m}$ de diámetro y con diámetro promedio de cerca de $0.1 \mu \mathrm{m}$, aproximadamente cien veces menor que el promedio de las partículas de cemento. El área superficial la microsílice condensada es aproximadamente 20,000 $\mathrm{m} 2 / \mathrm{kg}$.

El peso específico de la microsílice es generalmente de 2.20 a 2.5 , mientras que el peso específico del cemento portland es cerca de 3.15. El peso unitario de la microsílice varía de 130 a $430 \mathrm{~kg} / \mathrm{m} 3$.

La microsílice se vende en forma de polvo, pero es más comúnmente encontrado en la forma líquida. Se usa en cantidades que varían del $5 \%$ al $10 \%$ de la masa total de material cementante. Se emplea donde sea necesario un alto grado de impermeabilidad y alta resistencia del concreto. La microsílice sigue las normas ASTM C 1240 y NTP 334.087

Para la investigación se utilizará porcentajes de $5 \%$ a $10 \%$ del peso del material cementante para todas las relaciones $a /(c+p)$.

\section{Euco microsílica}

Es un aditivo en polvo listo para usarse en concreto. Este producto reacción a químicamente con el hidróxido de calcio en la pasta de cemento, lo cual produce un gel de hidrato de silicato de calcio que mejora de manera significativa la resistencia y durabilidad. Por su alta finura, la microsílica llena el vacío entre las partículas de cemento creando un concreto muy denso y menos permeable. Propiedades según Tabla 3 .

Tabla 3. Propiedad Euco microsílica

\begin{tabular}{|l|l|}
\hline Apariencia & Polvo gris de textura fina \\
\hline Gravedad Específica & 2.2 \\
\hline Densidad (granel) & Aprox. $481 \mathrm{~kg} / \mathrm{m}^{3}$ \\
\hline contenido de microsílica & $100 \%$ \\
\hline SiO2 Amorfo & $92-98 \%$ \\
\hline
\end{tabular}

Aplicaciones principales:

a. Concretos de altas resistencias

b. Concreto de alta densidad

c. Plataformas para puentes

d. Estructuras para estacionamientos

e. Ambientes marinos

f. Shotcrete.

g. Concretos de alta resistencia química.

\section{Características/Beneficios}

Altas resistencias a la compresión y a la flexión a todas las edades para una mejor capacidad estructural. Desarrollo de altas resistencias a edades tempranas para tiempos de ciclo de producción más cortos.

Le da al concreto una permeabilidad baja para una mejor resistencia a la penetración de agua y sal.

Mejor resistencia a la abrasión y a químicos para una mayor expectativa de vida/duración.

Mayores resistencias a descascararse y ciclos de hielo-deshielo.

Mejora el comportamiento del concreto, incrementa la producción y baja los costos de transporte y avance de obras

Aumenta la cohesión y reduce el lavado de finos.

\section{REDES NEURONALES ARTIFICIALES}

Una red neuronal está constituida por capas de neuronas. Una capa lo forman un conjunto de neuronas artificiales.

\section{Una Neurona Artificial}

Una neurona biológica tiene esencialmente tres componentes: el cuerpo, las dendritas que son los canales de recepción de señales que vienen de otras neuronas o del exterior y el axón que es el canal de emisión de la respuesta de una neurona.

Una neurona artificial es el elemento constitutivo de una RNA, recoge las características esenciales de una neurona biológica, está constituido por una matriz de pesos, un sesgo y una función de transferencia.

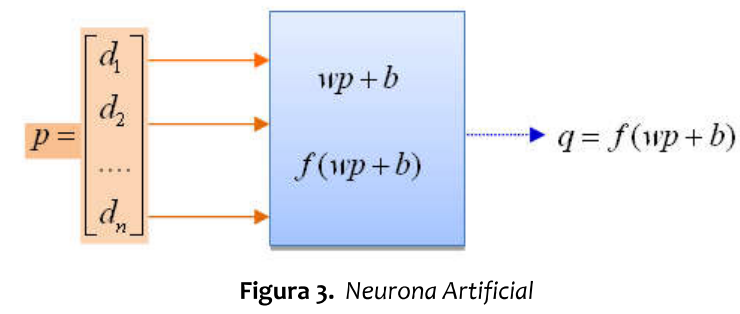

Los datos de ingreso a una neurona artificial, Figura 3 , está representado por un vector o matriz columna $\mathrm{P}=\left[d_{1}, d_{2}, \ldots, d_{\mathrm{n}}\right]$ donde cada componente 
representa una señal que al ingresar a la neurona es afectada por un número denominado peso. Esta afectación se representa por el producto $w_{k} d_{k}$. Una neurona tiene una matriz de pesos $w=\left[w_{1}, w_{2}, \ldots, w_{n}\right]$. El proceso en la neurona está representado por $w P+b=w_{1} d_{1}+w_{2} d_{2}+\ldots+w_{n} d_{n}+b$, donde es el sesgo de la neurona.

Los pesos expresan la incentivación o disminución de una señal por parte de la neurona, por ejemplo en $w_{1} d_{1}$ si $w_{1}$ toma valores grandes la señal $d_{1}$ está siendo incentivada, de lo contrario es reducida o se anulará cuando $w_{1}=0$. Luego interviene una función de transferencia $f(s)$ de la neurona, dando lugar a la respuesta final de la misma que es: $Q=f(w P+b)$

Existen muchas funciones de transferencia en el diseño de redes neuronales, entre ellas tenemos la tangente hiperbólica denominada tansig, la función identidad denominada purelin y otros.

\section{Una Capa de Neuronas Artificiales}

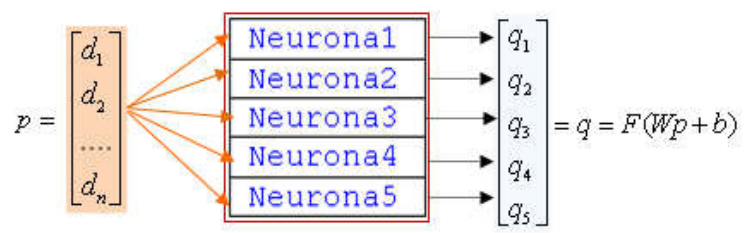

Figura 4. Capa de 5 Neuronas

Una capa de neuronas, Figura 4, está formada por $m$ neuronas, sin comunicación entre ellas, operan independientemente. Esto es, dispuestas en paralelo, $\mathrm{A}$ cada neurona $j$ le está asociada una matriz fila $W_{j}=\left[w_{j, 1}\right.$, $\left.w_{j, 2}, \ldots, w_{j, n}\right]$ que es la matriz de pesos de dicha neurona más un sesgo $b_{j}$ y una función de transferencia $f_{j}$.

Entonces si se denota con $W$ la matriz formada por las matrices fila de las $m$ neuronas y con $b$ la matriz columna de los sesgos, la acción de la capa de neuronas sobre un vector $P$ en una primera instancia es el producto y suma: $W P+b$, que es un vector de $\mathfrak{R}^{m}$.

En segunda instancia cada componente de $W P+b$ es transformada por la función de transferencia $f_{j}$ de la neurona. Este proceso se representa mediante una función vectorial a valores vectoriales: $\mathrm{f}: \mathfrak{R}^{\mathrm{m}} \rightarrow \mathfrak{R}^{\mathrm{m}}$ que a cada vector $u=\left(u_{1}, u_{2}, \ldots u_{m}\right) \in \mathfrak{R}^{m}$ le hace corresponder el vector $f(u)=\left[f_{1}\left(u_{1}\right), f_{2}\left(u_{2}\right), \ldots, f_{m}\left(u_{m}\right)\right]$. Entonces la acción de la capa de neuronas sobre el vector $P$ será:

$Q=f(W P+b)=\left[f_{1}\left(w_{1} P+b_{1}\right), f_{2}\left(w_{2} P+b_{2}\right), \ldots, f_{m}\left(w_{m} P+b_{m}\right)\right]$ que es un vector de $\mathfrak{R}^{\mathrm{m}}$.

Se dice que $Q=f(W P+b)$ es la respuesta o salida de la capa de neuronas para el vector de entrada $P$.
En consecuencia una capa de $m$ neuronas es una función $F: \mathfrak{R}^{n} \rightarrow \mathfrak{R}^{m}$ que transforma un vector $P \rightarrow \mathfrak{R}^{n}$ en otro $Q \in \mathfrak{R}^{m}$, donde $Q=F(P)=f(W P+b)$.

La observación pone en duda la estructura en paralelo de las redes neuronas, mostrando casos concretos de redes de neuronas interconectadas cumpliendo un protocolo de comunicación estructural que asegura la estabilidad del sistema de redes y no presenta fallas catastróficas.

\section{Una Red Neuronal Artificial}

Una red neuronal es una concatenación de capas de neuronas. Desde el punto de vista matemático es la composición de transformaciones:

$$
R^{n} \stackrel{F_{1}}{\longrightarrow} R^{k} \stackrel{F_{2}}{\longrightarrow} R^{S} \ldots \ldots \stackrel{F_{N}}{\longrightarrow} R^{m}
$$

\section{Redes Neuronales supervisadas: aprendizaje o entrenamiento}

Nos restringimos a las RNA para el estudio de la resistencia axial de las probetas con concreto de alta resistencia. En este caso se tiene 500 valores que son las resistencias de igual número de estas probetas $y$ que forman una matriz, que lo denominamos $T=\left[t_{1}, t_{2}, \ldots t_{M}\right]$, donde $t_{k}$ es la resistencia de la probeta cuyos datos de fabricación están registrados en la columna $p_{k}$, de la matriz $P=\left[p_{1}, p_{2}, \ldots p_{M}\right], p_{k} \in \mathfrak{R}^{n}$.

De otro lado, para cada la columna $p_{k}$ de $P$, una RNA devuelve un vector $q_{k}(x)$ donde $x$ es un vector que representa todos los pesos y sesgos de todas las neuronas que forman la red. De este modo la respuesta de la red para la matriz de datos $P$ que ingresa a ella, es otra matriz de números reales $Q(X)=\left[q_{1}(x), q_{2}(x), \ldots\right.$, $\left.q_{M}(x)\right]$.

El aprendizaje de la red consiste en conseguir un mínimo de la función:

$e(x)=\frac{1}{2} \sum_{k=1}^{M}\left(q_{k}(x)-t_{k}\right)^{2}$

Que es el error en media cuadrática entre $t=\left[t_{1}, t_{2}, \ldots, t_{M}\right]$ y la respuesta de la red $\mathrm{Q}(\mathrm{X})=\left[q_{1}(\mathrm{x}), \mathrm{q}_{2}(\mathrm{x}), \ldots, q_{\mathrm{M}}(\mathrm{x})\right]$.

Lo ideal es conseguir un $x *$ tal que $e\left(x_{*}\right)=0$, pero esto no siempre es posible, entonces lo que se hace es minimizar la función $e(x)$, buscando un $x$ * de tal manera que $\left\|e\left(x^{*}\right)\right\| \approx 0$. Esto implicará que para cada $\mathrm{k}=1,2, \ldots, \mathrm{M}$ la respuesta de la red $q_{\mathrm{k}}\left(\mathrm{x}^{*}\right)$ está muy próxima al valor esperado $t_{k}$. En este caso se dice que la 
RNA ha sido entrenada adecuadamente, en caso contrario no se habrá conseguido entrenar la red.

\section{ESTUDIO DEL CONCRETO DE ALTA RESISTENCIA}

\section{Ingreso de datos}

La base de datos es importada a MATLAB en forma de dos matrices: $P$ de 10 filas y 500 columnas, donde cada columna corresponde a los datos de fabricación de las probetas y $\mathrm{T}$ de una matriz fila 500 valores que son las resistencias de estas probetas.

En esta medida las columnas de $P$ son los vectores de entrada del espacio $R^{10}$ y $T$ es el valor esperado de la RNA y es un vector de 500 componentes.

\section{Separación de las columnas de $\mathrm{P}$ y los valores de $\mathrm{T}$ para la simulación}

Para esto se toma en cuenta que las columnas de la matriz $\mathrm{P}$ están numeradas del 1 a 500 . Entonces las columnas destinadas para la simulación de la red pueden ser seleccionadas empleando las clases de equivalencia módulo 5 ([1], [2]..., [5]= [0]) que ayuda a realizar esta selección de las columnas de $P$ y los valores correspondientes de $\mathrm{T}$, que reemplazan perfectamente a una selección aleatoria.

Se elegirán las columnas numeradas por la clase [5]. Esta clase será denotada: Clas5 y se define a continuación:

[fila col] $=\operatorname{size}(P)$;

Clas5 = 5:5:col; \%Tiene 99 miembros

Clasa $=[1: 5: \mathrm{col} 2: 5: \mathrm{col} 3: 5: \mathrm{col} 4: 5: \mathrm{col}]$;

$\mathrm{Pa}=\mathrm{P}(:$, Clasa $) ; \quad$ \% Para el entrenamiento

$\mathrm{Ta}=\mathrm{T}($ :,Clasa $)$;

$\mathrm{Pb}=\mathrm{P}($ :,Clas5); \%Para el Pronóstico

$\mathrm{Tb}=\mathrm{T}($ :,Clas5);

Normalización

$[$ pna,ps1] $=\operatorname{mapstd}(\mathrm{Pa})$

$[$ tna,ts] = mapstd $(\mathrm{Ta})$;

La función mapstd transforma las componentes $x$ de

cada fila de la matriz Pa en otra, empleando la fórmula es $H(x)=\frac{x-m c}{d c}$, donde $m c y d c$ son respectivamente, la media y la desviación estándar de la fila que contiene $a x$.

Este proceso transforma las filas de $\mathrm{Pa}$ y $\mathrm{Ta}$ en otras con media cero y desviación estándar 1.

Aquí se entiende que la matriz $\mathrm{Pa}$ ha sido transformada en pna y los elementos de la transformación que han intervenido en este proceso se encuentran en la clase ps1.

Similarmente cuando se hace mapstd(Ta) se produce el mismo efecto y se genera la clase ts.
Transformación de la dimensión de las columnas de pna

La segunda transformación que se hace es:

$$
\text { [ptransa,ps2] }=\operatorname{processpca}(p n a, 0.001) \text {; }
$$

En esta etapa al hacer processpca(pna, o.001) la matriz pna ha sido transformada en ptransa y los elementos de esta transformación se encuentran en la clase ps2.

El archivo ps2 contiene una transformación lineal (de $\mathrm{R}^{10}$ a $\mathrm{R}^{5}$ ) que transforma las columnas de pna, que son de dimensión 10 en otras de dimensión 5 , no elimina ninguna fila de pna. Esto se basa en que la matriz de covarianzas de la transpuesta de pna es de 10×10, simétrica, orto normal y como tal tiene 10 valores propios reales, de los cuales 5 son del orden de $10^{-14}$, es decir muy pequeños.

\section{Entrenamiento, Validación y Test de la RNA}

Para realizar estas tres fases se separan las columnas de ptransa en tres grupos disjuntos, donde cada grupo sea lo más variado posible de todo el universo de columnas. Esto se consigue empleando las 4 clases de equivalencia [1], [2], [3] y [4]. Para la fase del entrenamiento se toma la unión de la 1era y zera clase, para la validación la 4ta y para el Test la 2da clase. El proceso es como se indica a continuación:

[Fil colTa]=size $($ ptransa);

Clas13 = [1:4:colTa 3:4:colTa];

Clas4 = 4:4:colTa;

Clas2 = 2:4: colTa;

Enp = ptransa(:,Clas13);

VEt = tna(:,Clas13);

val.P = ptransa $(:$, Clas4 $)$;

val. $T=$ tna $(:$, Clas 4$)$;

test. $P=$ ptransa $(:$, Clasz $)$;

test. $T=$ tna $(:$, Clas2 $)$;

\section{Creación de la RNA Backpropagation (RNBP)}

Esta red se denominará "net", será de 4 capas, con 5 neuronas la primera (que NO coincide con el número de filas o variables de ptransa que son 5) con 15 neuronas la segunda, con 10 la tercera y 1 neuronas en la capa de salida, pues el valor esperado tna es una matriz de $1 \times 500$. Las funciones de transferencia son tansig para las tres primeras capas y purelin para la última capa.

Rango=minmax (pna);

net $=$ newff(Rango, $\left[\begin{array}{llll}5 & 15 & 10 & 1\end{array}\right],\{$ 'tansig' 'tansig' 'tansig' 'purelin'\},'trainlm'); [net, tr] =train(net,Enp,VEt, [],[],val,test); 
Luego de ejecutar el programa anterior se tiene resultados como Interfase Figura 5

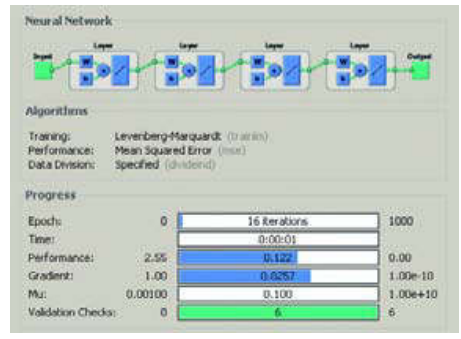

Figura 5. Interface

\section{Performance (gradiente)}

En ambas gráficas se visualiza el comportamiento del gradiente en la búsqueda del mínimo local de una función no lineal de 555 variables, que son los pesos y sesgos de todas las neuronas que conforman la red neuronal "net" que se ha creado.

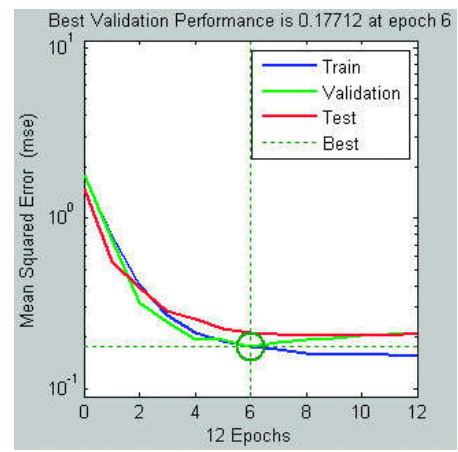

Figura 6. Resultados RNA Backpropagation

\section{Correlación}

Los resultados del entrenamiento alcanzan una correlación de 0.92334 , la validación 0.89653 y el test o prueba de a una correlación de 0.8680 y la correlación total es de 0.9036 . Figura 7

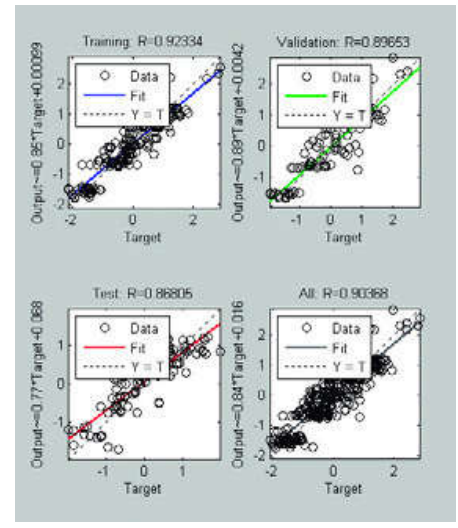

Figura 7. Correlación final

\section{Simulación}

a. Respuesta de la RNBP net con datos que ingresaron para su entrenamiento

Esto es con la finalidad de ver cuáles son los valores de la RNBP que entrega luego de haberse entrenado y recibido como input la matriz pnr que es la transformación de pn y este es a su vez la transformación de pa. Pero al final se quiere saber cuál es la salida de la RNBP creada y entrenada, que se denotará qa. Figura 8

$r a=\operatorname{sim}($ net, ptransa);

$R a=$ mapstd('reverse',ra,ts);

b. Correlación entre Ra y Ta

$[m, b, r]=\operatorname{postreg}(R a, T a)$;

$[\mathrm{m}, b, r]$

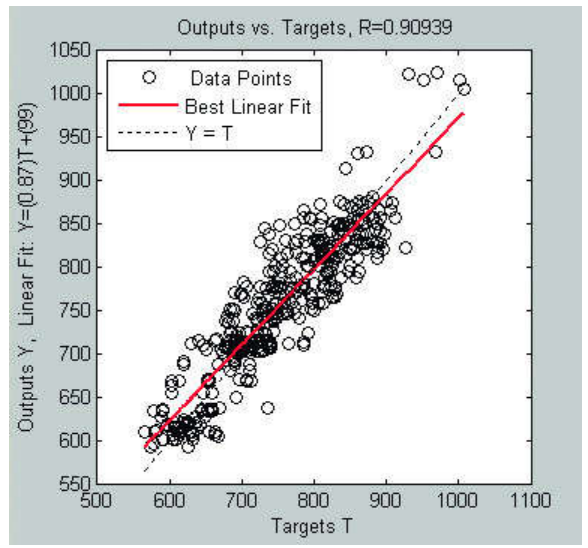

Figura 8. Resultados Reales y Estimado

ans $=\begin{array}{lll}0.8012 & 154.2150 & 0.9038\end{array}$

Respuesta de pendiente e intercepto de regresión y la correlación de valores reales y estimados.

\section{Estadísticas de los resultados}

a. Coeficientes de determinación: $R, R^{2}, M S E$ (error en m. c.) y RMSE (raíz). Ver Tabla 4.

$\mathrm{Na}=$ length $(\mathrm{Ra})$;

$m R=\operatorname{mean}(R a)$;

$m T=\operatorname{mean}(T a)$;

$V m R=m R^{*}$ ones $(1, \mathrm{Na})$;

$V m T=m T^{*}$ ones $(1, \mathrm{Na})$;

$c R=\operatorname{dot}(R a-V m R, T a-V m T) /(\operatorname{norm}(R a-V m R) *$

$\operatorname{norm}(T a-V m T))$;

$M S E=\operatorname{norm}(\mathrm{Ta}-\mathrm{Ra}, 2)^{\wedge} 2 / \mathrm{Na}$;

RMSE = norm $(\mathrm{Ta}-\mathrm{Ra}, 2) / \mathrm{sqrt}(\mathrm{Na})$;

DATOSESTADIS $a=\left[c R, c R^{\wedge} 2, M S E, R M S E\right]$ 
Tabla 4. Resultado de DATOSESTADIS

\begin{tabular}{|c|c|c|c|c|}
\hline ESTRUCTURA & $R$ & $\mathrm{R} 2$ & MSE & RMSE \\
\hline $\begin{array}{llll}5 & 15 & 10 & 1\end{array}$ & 0.912 & 0.833 & 1328 & 36.44 \\
\hline
\end{tabular}

b. Creación de la Tabla con Ta y Ra ordenadas Ordenando Ta juntamente con Ra de menor a mayor. Ver Figura 9.

[filaTa colTa] = size $(T a)$;

$J a=1: 1:$ colTa; \% Contador

Aux = Ta;

for $i=1$ : colTa

$[a, i x]=\min (A u x)$;

$\mathrm{TaO}(\mathrm{i})=a$;

$\operatorname{Aux}(i x)=\ln f ;$

$\operatorname{RaO}(i)=R a(i x)$;

end

$\mathrm{Ea}=\mathrm{abs}(\mathrm{TaO}-\mathrm{RaO}) ; \%$ (Error);

Gráficas de $\mathrm{TaO}$ juntamente con RaO y en Lila el error Ea

plot(Ja,RaO,'or','MarkerSize',5,'MarkerFaceColor', [.291.60]);

hold on;

plot(Ja,TaO, 'ob','MarkerSize',5, 'MarkerFaceColor',

[.49 1.63]);

plot(Ja, Ea, 'om','MarkerSize',5,'MarkerFaceColor',

$\left.\left[\begin{array}{ll}.29 & 1.25\end{array}\right]\right)$;

xlabel('NUMERO DE ITERACIONES');

ylabel('VALORES Ra y Ta ORDENADOS');

legend('Ra=RESPUESTA DE LA RED ', 'Ta=RESISTENCIA

DEL CONCRETO', 'Ea=|Ta-Ra|= ERROR');

title('RESPUESTA DE LA RED Ra VS VALOR ESPERADO Ta');

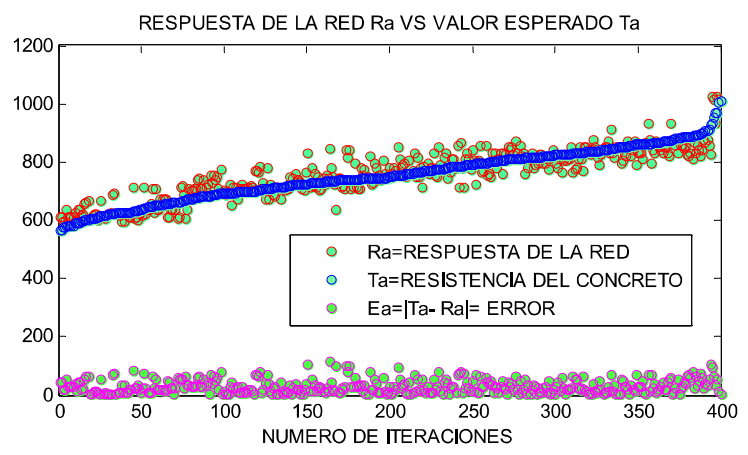

Figura 9. Valores Reales, Estimados y Error

Azul= Valor esperado $T a$

Rojo=Salida de la Red $R a$

Lila=Error $E a=|T a-R a|$

\section{Capacidad predictiva de la RNBP}

a. Respuesta de la red para los datos $\mathrm{Pb}$ y Tb En esa oportunidad se creó la clase psz que contiene una transformación lineal que reduce la dimensión de las columnas que ingresan a la red. Entonces se tiene:

$[p n b, p s 3]=\operatorname{mapstd}(\mathrm{Pb})$;

Trans = ps2.transform;

ptransb = Trans*pnb;

$r b=\operatorname{sim}$ (net, ptransb);

$R b=$ mapstd('reverse',rb,ts);

$E b=a b s(R b-T b)$;

Ib = 1:1:length $(R b) ; \%$ Contador

$T A B L A b=\left[I b^{\prime}, R b^{\prime}, T b^{\prime}, E b^{\prime}\right]$;

La correlación entre $\mathrm{Rb}$ y $\mathrm{Tb}$, se tiene corriendo el siguiente programa:

$[m, b, r]=\operatorname{postreg}(R b, T b)$;

$[\mathrm{m}, b, r]$

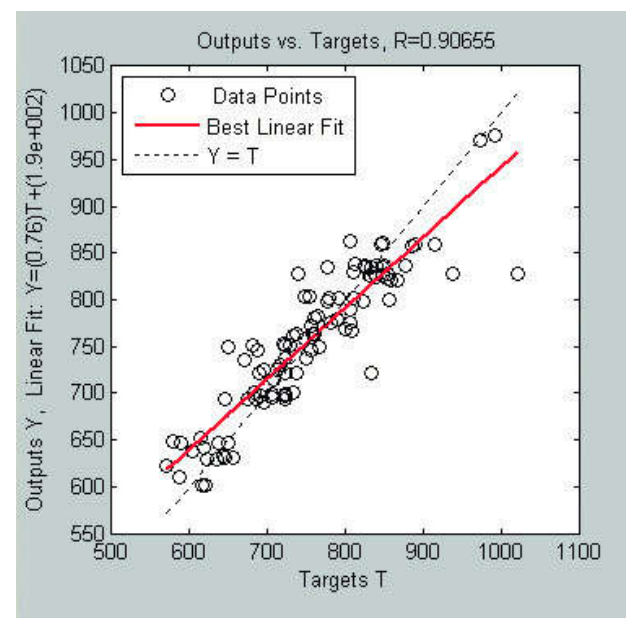

Figura 10. Resultados de Valores reales y predicción

ans $=0.7565 \quad 186.1142 \quad 0.9065$

Donde $\boldsymbol{m}=0.7565, \boldsymbol{b}=186.1142$ son la pendiente y la intersección de la recta con el eje Y. La correlación $\mathbf{r}=$ 0.9065 entre la respuesta y el valor esperado de la red. Ver Figura 10.

b. Coeficientes de determinación: $R, R^{2}, M S E$ y RMSE para Rb y Tb. Ver Tabla 5.

$N b=$ length $(R b)$;

$m R=\operatorname{mean}(R b)$;

$m T=\operatorname{mean}(T b) ;$

$V m R=m R^{*}$ ones $(1, N b)$;

$V m T=m T^{*}$ ones $(1, N b)$;

$c R=\operatorname{dot}(R b-V m R, T b-V m T) /(\operatorname{norm}(R b-V m R)$ *

norm $(T b-V m T))$;

$M S E=\operatorname{norm}(\mathrm{Tb}-\mathrm{R} b, 2)^{\wedge} 2 / \mathrm{Nb}$;

RMSE = norm $(T b-R b, 2) / \operatorname{sqrt}(\mathrm{Nb})$;

DATOSESTADISb $=\left[C R, c R^{\wedge} 2, M S E, R M S E\right]$ 
Tabla 5. DATOS ESTADIS

\begin{tabular}{|llll|c|c|c|c|}
\hline \multicolumn{3}{|c|}{ ESTRUCTURA } & $\mathbf{R}$ & $\mathbf{R}^{\mathbf{2}}$ & MSE & RMSE \\
\hline 5 & 15 & 10 & 1 & 0.912 & 0.833 & 1328 & 36.44 \\
\hline 20 & 14 & 10 & 1 & 0.909 & 0.826 & 1392 & 37.31 \\
\hline 25 & 15 & 5 & 1 & 0.864 & 0.746 & 1991 & 44.62 \\
\hline 2 & 4 & $6 S$ & 1 & 0.873 & 0.763 & 1866 & 43.19 \\
\hline
\end{tabular}

c. Comparación gráfica del pronóstico y el valor esperado

Ordenando $\mathrm{Tb}$ y $\mathrm{Rb}$ de menor a mayor $\mathrm{y}$ graficando. Ver Figura 11.

[filaTb colTb] = size $(\mathrm{Tb})$;

$J b=1: 1:$ colTb; \% Contador

Aux $=\mathrm{Tb}$;

for $i=1:$ colTb

$[b, i x]=\min (A u x)$;

$\mathrm{TbO}(\mathrm{i})=b$;

Aux(ix) = Inf;

$R b O(i)=R b(i x)$;

end

$E b=a b s($ TbO-RbO); \% (Error);

Gráficas de TbO juntamente con RbO y en Lila el error Eb

plot(Jb, RbO, 'or', 'MarkerSize',5, 'MarkerFaceColor', [.29 1.60]);

hold on;

plot(Jb, TbO, 'ob','MarkerSize',5,'MarkerFaceColor', [.49 1.63]);

plot(Jb, Eb, 'om','MarkerSize',5,'MarkerFaceColor', $\left.\left[\begin{array}{ll}.29 & 1.25\end{array}\right]\right)$; xlabel('NUMERO DE ITERACIONES');

ylabel('VALORES Rb y Tb ORDENADOS');

legend('Ra=RESPUESTA DE LA RED ', 'T $b=$ RESISTENCIA DEL CONCRETO', 'Eb=|Tb-Rb|=ERROR');

title('PRONOSTICO DE LA RED Rb VS VALOR ESPERADO $\left.T b^{\prime}\right)$;

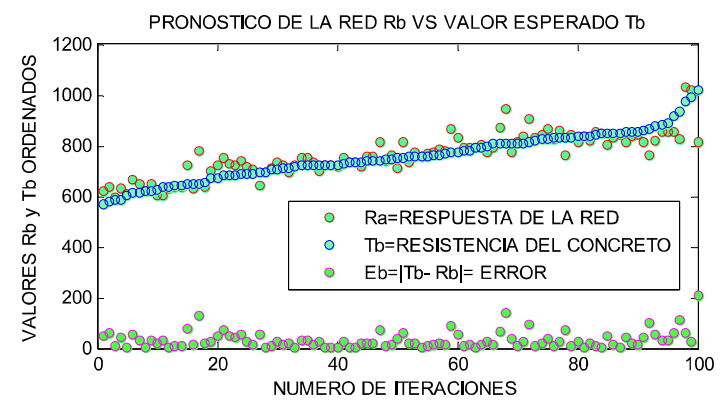

Figura 11. Valores Reales, Estimados y Error

\section{RESULTADOS}

Los coeficientes de determinación obtenidos en el conjunto de comprobación $\mathrm{Pb}$ indican que la RNBP desarrollada consigue hacer un pronóstico del 90\% de la totalidad de las probetas consideradas.

Los resultados obtenidos durante el proceso de desarrollo de la red, con coeficientes de determinación obtenidos $\left(R=0.912\right.$ y $\left.R^{2}=0.83\right)$ son superiores a los obtenidos por Yeh [18] y por Ozturan et al. [12] y Prasad et al. [13] los cuales obtuvieron coeficientes máximos entre 0.78 y 0.92 .

Yeh [18] y Ozturan et al. [12] también modelizaron la resistencia del concreto mediante modelos de regresión. Sin embargo, obtuvieron resultados inferiores a los obtenidos en este estudio. Así, para dichos modelos, Yeh [18] obtuvo coeficientes de determinación entre 0.46 y 0.89 y Ozturan et al. [12] obtuvieron coeficientes de determinación entre $0.55 \mathrm{y}$ 0.79 .

\section{AGRADECIMIENTOS}

Este trabajo fue desarrollado dentro del Proyecto de Investigación Proyecto PIAP-1-P-676-13 financiado por FINCyT, dentro del Programa de Proyectos de Investigación Aplicada 2013. 


\section{REFERENCIAS}

[1] ASTM C 192/C 192M: The American Society for Testing Materials Standard Practice for Making and Curing Concrete Test Specimens in the Laboratory, West Conshohocken (PA), USA (2000).

[2] ASTM C 39/C 39M: The American Society for Testing Materials, Standard Test Method for Compressive Strength of Cylindrical Concrete Specimens, West Conshohocken (PA), USA (2001)

[3] Caldarone, M.A., High-Strength Concrete: A Practical Guide, Taylor and Francis, New York, USA (2009).

De Veaux R.D. y L.G. Ungar, A brief introduction to neura networks, (1996).

[4] Demuth, H., M. Beale y M. Hagan, Neural Network Toolbox User's Guide, Version 4, The MathWorks Inc., Natick, USA (2002).

[5] Fernández, F., P. de Palacios, L. García Esteban, A. García-Iruela, B. González Rodrigo y E. Menasalvas, Prediction of MOR and MOE of structural plywood board using an artificial neura network and comparison with a multivariate regression model, Composites: Part B: 43, 3528-3533 (2012).

[6] Hornik, K., Multilayer Feedforward Networks are Universal Approximators, Neural Networks: 2, 359-366 (1989). http://www.cis.upenn.edu/ ungar/Datamining/Publications/ nnet-intro.pdf. Acceso: 26 de mayo (2014).

[7] Kosmatka, S.H., B. Kerkhoff y W.C. Panarese, Design and Control of Concrete Mixtures, Portland Cement Association. Skokle, Illinois, USA (2003).

[8] Lee, S.C., Prediction of concrete strength using artificial neural networks, Journal of Engineering Structure: 25, 849-857 (2003).

[9] Lin T.Y. y C.H. Tseng, Optimum design for artificial networks: an example in a bicycle derailleur system, Engineering Application of Artificial Intelligence: 13, 3-14 (2000).
[10] Ozerdem, M.S. y S. Kolukisa, Artificial neural network approach to predict the mechanical properties of $\mathrm{Cu}-\mathrm{Sn}-\mathrm{Pb}-\mathrm{Zn}-\mathrm{Ni}$ cast alloys, Materials and Design: 30, 764-769 (2009).

[11] Oztas, A., M. Pala, E. Ozbay, E. Kanka, A. Caglar y M.A. Bhatti, Predicting the compressive strength and slum of high strength concrete using neural network, Construction and Building Materials: 20-9, 769-775 (2006)

[12] Ozturan, M., B. Kutlu, y T. Ozturan, Comparison of concrete strength prediction techniques with artificial neural network approach, Building Research Journal: 56, 23-36 (2008).

[13] Prasad, B.K.R., H. Eskandari y B.V.V. Reddy, Prediction of compressive strength of SCC and HPC with hay volumen fly ash using ANN, Construction and Building Materials: 23, 117-128 (2009).

[14] Reddy, N.S., J. Krishnaiah, S.G. Hong y J.S. Lee, Modeling medium carbon steels by using artificial neural networks, Materials Science and Engineering A: 508, 93-105 (2009).

[15] Ukrainczyk, N. y V. Ukrainczyk; A neural network method for analysing concrete durability, Magazine of Concrete Research: $60(7), 475-486$ (2008).

[16] W. y J. Tannock, The training of neural networks to model manufacturing processes, Journal of Intelligent Manufacturing: 16, 39-51 (2005).

[17] Yaprak, H., A. Karaci, y I. Demir, Prediction of the effect of varying cure conditions and $w / c$ ratio on the compressive strength of concrete using artificial neural networks, Neural Computing and Applications: 22, 133-141 (2013).

[18] Yeh, I.-C.,Modeling of strength of high-performance concrete using artificial neural networks, Cement \& Concrete Composites, 28 (12), pp. 1797-1808 (1998) 